EPJ manuscript No.

(will be inserted by the editor)

\title{
Physics at ELSA
}

\author{
Hartmut Schmieden ${ }^{1}$ and the Crystal-Barrel/TAPS collaboration \\ Physikalisches Institut, Rheinische Friedrich-Wilhelms-Universität Bonn \\ Received: date / Revised version: date
}

\begin{abstract}
Recent experimental results of the Crystal-Barrel/TAPS collaboration at ELSA are presented. The experiments used a tagged photon beam incident on proton and neutron targets. Multiple photon final states were detected in the hermetically closed Crystal Barrel/TAPS detector setup, enabling the reconstruction of various neutral meson production channels. In addition to cross sections also photon beam asymmetries and, in the $K_{s}^{0} \Sigma^{+}$channel, recoil polarisations were determined.
\end{abstract}

PACS. 13.60.-r Photon and charged-lepton interactions with hadrons - 13.60.Le Meson production 13.88.+e Polarization in interactions and scattering - 14.20.Gk Baryon resonances with $\mathrm{S}=0$

\section{Introduction}

The rich excitation spectrum of the nucleon reflects its complicated inner dynamics. Hence baryon spectroscopy is expected to provide benchmark data for any model of the nucleon, e.g. quark models in their variety [1,2] or, increasingly in the near future, Lattice QCD as an approximation of full Quantum Chromodynamics 3. The major problem of nucleon spectroscopy is the width and density of states involved which, in many cases, prohibit their clean identification, i.e. an unambiguous assignment of quantum numbers within a partial wave analysis.

The current experimental base is mostly restricted to pion and kaon induced reactions. However, a significant number of excited states is suspected to have a strongly disfavoured $\pi N$ coupling [4. Hence, such states might have escaped detection in conventional analyses. This is one possible scenario why much less baryonic excitations are observed than expected by quark models and provided the motivation to investigate photoinduced reactions beyond single-pion production [5, 6,7]. In particular, we studied $\eta$ photoproduction off proton and deuteron targets, two-meson final states, associated strangeness photoproduction in the $K_{s}^{0} \Sigma^{+}$channel, and $\omega$ photoproduction.

Unambiguous solutions of partial wave analyses are generally only possible on the basis of "complete" experiments with regard to the separation of reaction amplitudes. Such measurements appear at the horizon in $\eta$ and $K$ photoproduction. In these cases the required number of, if carefully chosen, 8 independent observables $[8$, seems accessible. In two-meson and vectormeson photoproduction the measurement of the required 16 or 23 quantities is presently out of range. Nevertheless, polarisation observables provide essential constraints which, if not to isolate specific resonances, still may allow to clarify the reaction mechanism regarding whether s-channel resonances contribute at all.

Such experiments depend on linearly and circularly polarised photon beams. The measurement of double polarisation observables, inescapable for the "complete" experiment, require polarised nucleon targets in addition. Eventually, the recoil nucleon polarisation needs to be determined as well.

In the following section some of the observables of meson photoproduction are discussed. Section 3 then describes the experimental setup before in section 4 some recent, partially preliminary, results are discussed. The final section summarises and gives an outlook to upcoming experiments.

\section{Cross section and polarisation observables}

With polarised beam and target, in the simplest case of photoproduction of single pseudoscalar mesons the cross section can be written in the form [9]

$$
\begin{aligned}
\frac{d \sigma}{d \Omega}=\frac{d \sigma_{0}}{d \Omega}[1 & -P_{\operatorname{lin}} \Sigma \cos 2 \Phi \\
& +P_{z}\left(-P_{\operatorname{lin}} H \sin 2 \Phi+P_{\text {circ }} F\right) \\
& -P_{y}\left(-T+P_{\operatorname{lin}} P \cos 2 \Phi\right) \\
& \left.-P_{z}\left(-P_{\text {lin }} G \sin 2 \Phi+P_{\text {circ }} E\right)\right],
\end{aligned}
$$

where $\sigma_{0}$ denotes the polarisation independent cross section, $P_{\text {lin, circ }}$ the degree of linear or circular polarisation of the incident photon beam, and $\Phi$ the azimuthal orientation of the reaction plane with respect to the plane of linear polarisation. The photon direction defines the $z$ axis of a right handed coordinate frame spanned with the outgoing meson momentum $\boldsymbol{k}_{m}: \boldsymbol{z}=\boldsymbol{k}_{\gamma} /\left|\boldsymbol{k}_{\gamma}\right|, \boldsymbol{y}=\boldsymbol{k}_{m} /\left|\boldsymbol{k}_{m}\right|$, and $\boldsymbol{x}=\boldsymbol{z} \times \boldsymbol{y} . P_{x, y, z}$ are the cartesian components of 
Hartmut Schmieden, the Crystal-Barrel/TAPS collaboration: Physics at ELSA

the target polarisation vector in this frame. Once beam and/or target are polarised, the polarisation observables $\Sigma, H, F, T, P, G$ and $E$ are accessible. In particular, the measurements require the following combinations of beam and target polarisation:

$\begin{array}{cll}\text { observable } & \text { beam } & \text { target } \\ \Sigma & \text { linear } & \text { no } \\ T & \text { no } & \text { transverse } \\ H & \text { linear } & \text { transverse } \\ F & \text { circular } & \text { transverse } \\ P & \text { linear } & \text { transverse } \\ G & \text { linear } & \text { longitudinal } \\ E & \text { circular } & \text { longitudinal }\end{array}$

The polarisation observables represent ratios of structure functions and as such are related to the more general case of meson electroproduction. Although more involved than photoproduction, through variation of the momentum transfer electroproduction is able to characterise the spatial structure of the processes involved [10] in addition to the time structure which is obtained through the spectroscopic information with real photons. If instead of, or, in addition to target polarisation the measurement of nucleon recoil polarisation is provided, further double and triple polarisation observables can be defined which, with their mutual interrelations, are discussed in detail in [9].

In the sense of the "complete" experiment mentioned in the introduction, it is necessary to determine angular distributions of at least 8 quantities. Those must encompass the differential cross section and the single polarisation observables $\Sigma$ and $T$, as well as the recoil polarisation. Furthermore, 4 double polarisation, e.g. beam-target, observables need to be chosen such that the occurence of discrete ambiguities can be avoided in the analysis of the bilinear forms of the reaction amplitudes 8 .

The situation becomes more complicated in the photoproduction of double pseudoscalars 11] and vector mesons. Despite the infeasibility of "complete" experiments with current techniques in such cases, essential information towards the involved reaction mechanisms, in particular the role of s-channels resonances, can be expected from the investigation of single and double polarisation observables.

The understanding of the reaction mechanisms is a prerequisite for partial wave analyses (PWA) or dynamical models to disentangle the broad and overlapping states. Different final states and concurring (coupled) channels need to be investigated as well as proton and neutron targets. In addition to polarisation, also full coverage of the angular and energy range is essential. Thus, the combination of the Crystal Barrel [12] and TAPS [13] detectors to an almost $4 \pi$ array at the Bonn electron stretcher accelerator ELSA [14] provides an ideal tool for the sketched investigations.

\section{Experimental setup}

The experiments were performed at the tagged photon beam of ELSA. Electron beams up to $E_{0}=3.5 \mathrm{GeV}$ were

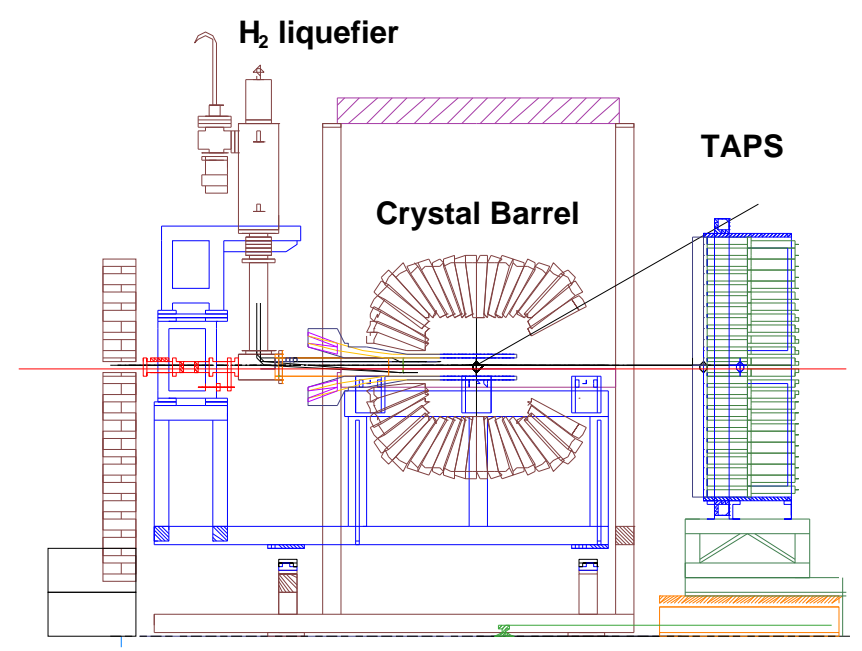

Fig. 1. Setup of the detector system as described in the text. The photon beam enters from left.

used to produce unpolarised or linearly polarised [15, 16 bremsstrahlung. Electrons which radiated a photon were momentum analysed in a tagging spectrometer, enabling the event-wise assignment of the photon energy in the range $E_{\gamma}=0.18 \ldots 0.92 E_{0}$. Photon fluxes of about $2 \times 10^{7}$ $\mathrm{s}^{-1}$ were usually used.

The detector setup is depicted in Figure1 The photon beam hit a $5.3 \mathrm{~cm}$ long liquid hydrogen or deuterium target. A three layer scintillating fibre detector [17], which surrounded the target within the polar angular range from $15-165$ degrees, was used to determine a point-coordinate for charged particles.

Both, charged particles and photons were detected in the Crystal Barrel detector [12. Its 1290 individual CsI(Tl) crystals were cylindrically arranged around the target in 23 rings, covering a polar angular range of $30-168$ degrees. For photons an energy resolution of $\sigma_{E_{\gamma}} / E_{\gamma}=$ $2.5 \% / \sqrt[4]{E_{\gamma} / \mathrm{GeV}}$ and an angular resolution of $\sigma_{\Theta, \Phi} \simeq$ 1.1 degree was obtained.

The 5.8 - 30 degree forward cone was covered by the TAPS detector [13, set up in one hexagonally shaped wall of $528 \mathrm{BaF}_{2}$ modules at a distance of $118.7 \mathrm{~cm}$ from the target. For photons between 45 and $790 \mathrm{MeV}$ an energy resolution of $\sigma_{E_{\gamma}} / E_{\gamma}=\left(0.59 / \sqrt{E_{\gamma} / \mathrm{GeV}}+1.9\right) \%$ was achieved [18. The position of photon incidence could be resolved within $20 \mathrm{~mm}$. For charged particle recognition each TAPS module had a $5 \mathrm{~mm}$ plastic scintillator in front of it.

\section{Recent Results}

The combined Crystal Barrel and TAPS setup is ideally suited for final states of multiple photons. Hence, neutral mesons are very efficiently detected. Those are of particular interest for nucleon spectroscopy since t-channel exchanges, which may hide resonance excitations, are suppressed in many cases. Investigated channels involve $\pi^{0} \rightarrow$ 
$\gamma \gamma, \eta \rightarrow \gamma \gamma$ or $3 \pi^{0}, K_{s}^{0} \rightarrow \pi^{0} \pi^{0} \rightarrow 4 \gamma$, and $\omega \rightarrow$ $\pi^{0} \gamma \rightarrow 3 \gamma$, as well as combinations thereof. Typical invariant mass resolutions achieved were $\sigma_{\pi^{0}}=10 \mathrm{MeV}$ and $\sigma_{\eta}=22 \mathrm{MeV}$ in the two-photon decays of $\pi^{0}$ and $\eta$, and $\sigma_{\eta}=25 \mathrm{MeV}$ in $\eta \rightarrow 3 \pi^{0}$.

\section{1 $\eta$ photoproduction off the proton}

Similar to pion photoproduction by the $\Delta(1232)$ resonance so is $\eta$ photoproduction in the threshold region dominated by a single nucleon resonance, the $S_{11}(1535)$. Vice versa, $\eta$ photoproduction gives very selective access to this state, the internal structure of which is still under debate. Furthermore, $\eta$ photoproduction is a predestined channel to look for possible resonances with small $\pi$-N coupling which might have escaped experimental detection to date. Due to its isoscalarity, the $\eta$ only connects $N^{*}$ as opposed to $\Delta^{*}$ resonances with the nucleon ground state, which simplifies the observed spectrum considerably.

The role of resonances beyond the $S_{11}$ is, due to its dominance, much less clear. The strategy to investigate this at ELSA was threefold: First, differential cross sections have been determined over the full angular range across the full nucleon resonance region. Second, as a first step towards the "complete" experiment the photon beam asymmetry has been measured at the high-energy tail of the $S_{11}$. And third, neutron targets have been included in the investigations as will be discussed later.

A recent cross section measurement of the Crystal Barrel Collaboration at ELSA provided some indication for a yet unobserved $N^{*}(2070) D_{15}$ state [5,19]. The data agree well with the CLAS data which are available at somewhat lower energy [20, but both data sets are normalised only relative to the SAID $\pi^{0}$ cross section. This deficiency could now be remedied by a detailed analysis of the photon flux based on the timing and rate information of the tagging system 21. The preliminary result for one energy bin is shown in Figure [2 In addition to the absolute normalisation, the full data set, which presently is in the final analysis stage, has also improved angular coverage in forward direction as well as improved statistics compared to the published data of ref. [19].

Using a linearly polarised photon beam, the photon beam asymmetry $\Sigma$ was measured [15,16] based on the azimuthal modulation of the cross section (cf. Eq.11). The results agree well with the measurements at GRAAL [22, 23 . In the light of the introductory discussion the measurement of $\Sigma$ is absolutely indispensable to disentangle the resonances possibly contributing to the reaction. The analysis of the new Crystal Barrel data within the MAID isobar model 24 and the Bonn-Gatchina PWA 25] showed, however, that no unambiguous conclusion can yet be drawn. Both MAID and the Bonn-Gatchina partial wave analysis provide a satisfactory overall description of our data. In detail, however, there are marked differences with regard to the role of individual resonance contributions, cf. the figures and detailed discussion in [15, 16.

To resolve this problem, further double polarisation experiments are indispensable.

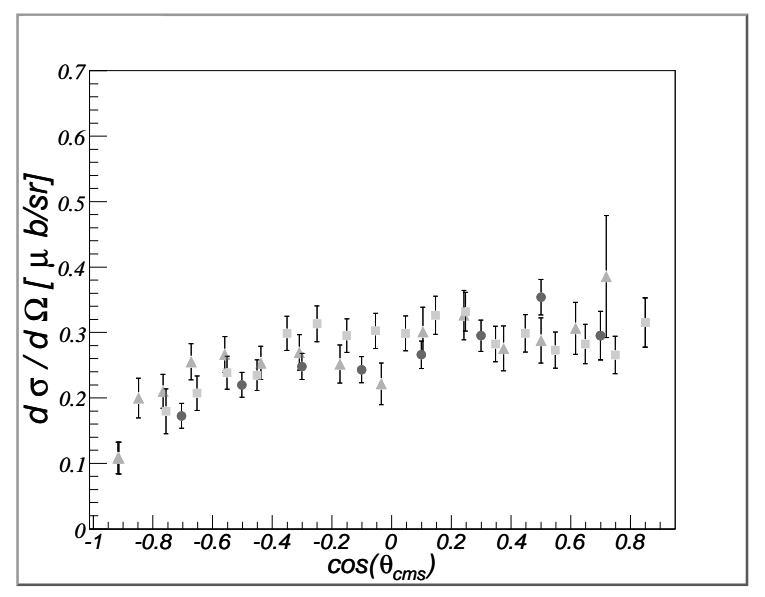

Fig. 2. Preliminary differential cross section with absolute normalisation of Crystal Barrel/TAPS 21. (triangles) in the bin $E_{\gamma}=(1075 \pm 25) \mathrm{MeV}$ in comparison to earlier measurements from Crystal Barrel [19] (squares) and CLAS 20] (circles) which are normalised relative to the SAID $\pi^{0}$ photoproduction database.

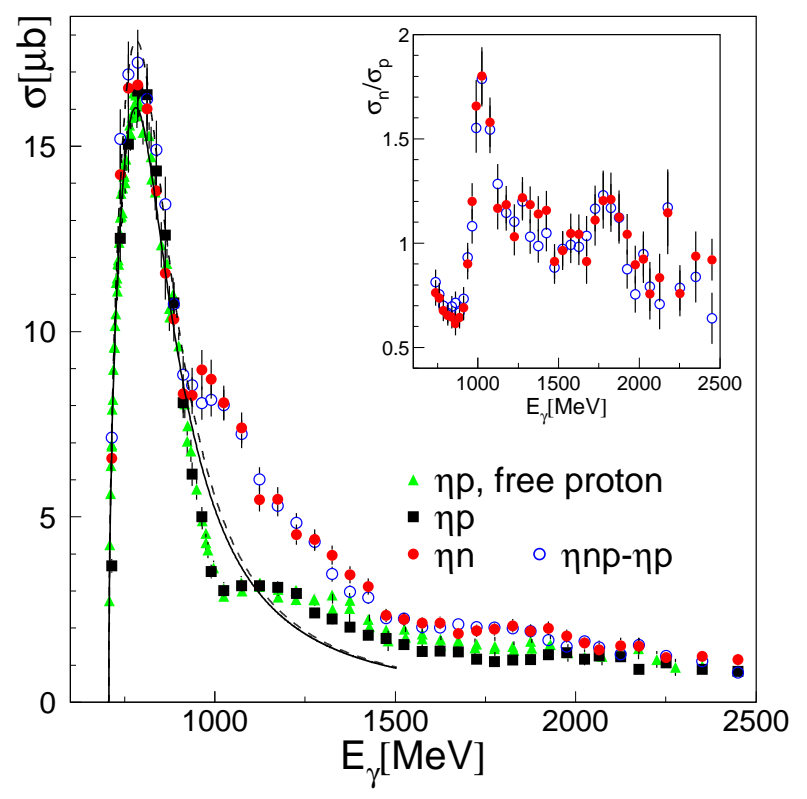

Fig. 3. Preliminary result for the total cross sections of $\eta$ photoproduction off the deuteron. Squares denote the quasifree $\eta$ production off the bound proton, filled circles off the neutron. The Fermi smearing has been unfolded. The open circles represent the difference between total quasifree production off the deuteron and the elementary production of the free proton. The latter is individually shown by the triangles. The insert depicts the ratio of neutron over proton cross section.

\section{$4.2 \eta$ photoproduction off the neutron}

Off the neutron, $\eta$ photoproduction had attracted a lot of activity recently. The reason is a rather narrow structure which seemed to exhibit itself in the cross section [26]. Also in the preliminary Crystal Barrel/TAPS data which are presented in Figure 3 there is a clear structure visible 
in the neutron cross section at around $E_{\gamma} \simeq 1100 \mathrm{MeV}$ [27. Such a bump is present neither in the free nor the quasifree proton cross section. This had triggered speculations about the influence of a narrow (antidecuplet) state [29]. However, conventional explanations are also possible, albeit the nature of the structure still remains under debate.

Interferences within the $S_{11}$ partial waves, e.g. between $S_{11}(1535)$ and $S_{11}(1650)$, seem to play a crucial role, both within the Bonn-Gatchina PWA 30 and the Giessen coupled channels model 31]. Interferences between different partial waves, $S_{11}(1535)$ and $D_{13}(1520)$ are under discussion, do exhibit in the angular distributions, but those can not affect the total cross section. The direct contribution of the $D_{13}(1520)$ is much too small to become responsible for the bump. This may be different for the $D_{15}(1675)$ state. Within the MAID model it has a particularly strong coupling to the neutron, at the same time however also an unusal nucleon- $\eta$ decay branch of $17 \%$, in disagreement with the PDG value of $0-1 \%$. To clarify this still unsatisfactory situation, measurements of $\Sigma, E$ and $G$ are planned [28.

\section{$4.3 \mathrm{~K}_{\mathrm{s}}^{0} \Sigma^{+}$photoproduction}

There are substantial decay branches of baryon resonances expected into $K$-hyperon channels 32,33 . Hence, associated strangeness photoproduction is also a channel where so far unoberved states of the quark models may be found. The reaction mechanism should become disentangled once the complete experiment is approached with regard to the reaction amplitudes. To achieve this, the $K \Lambda / \Sigma$ channels are very well suited due to the self analysing weak decay of the hyperons. This facilitates the indispensable measurement of the recoil polarisation.

The $K^{0} \Sigma^{+}$channel has been investigated with Crystal Barrel/TAPS from threshold to $W=2.3 \mathrm{GeV}$. In a first step cross sections and recoil polarisation were determined [34 and compared to calculations within the BonnGatchina PWA 25, 36 and a coupled-channels K-matrix formalism of Usov and Scholten 37. The total cross section is well reproduced by both calculations. However, an additional new state at about $1840 \mathrm{MeV}$ is required which in the PWA analysis has $P_{11}$ quantum numbers, in contrast to $P_{13}$ in the Usov-Scholten model. Such an ambiguity is no surprise given the "incompleteness" of the present experiments. It will be remedied with the availability of polarisation observables.

The first measurement of the recoil hyperon polarisation with Crystal Barrel/TAPS 34 has been statistically improved in the meanwhile and, in addition, the photon beam asymmetry has been measured [35]. Preliminary results are shown in Figure 4 .

\subsection{Two pseudoscalar meson channels}

Double meson final states provide a tool to get access to high-lying excitations which decay sequentially. Data of $\pi^{0} \pi^{0}$ and $\pi^{0} \eta$ final states were taken over the entire resonance region $42,43,44$.

From the $2 \pi^{0}$ channel partial decay widths of $N^{*}$ and $\Delta^{*}$ decaying into $\Delta(1232) \pi, N(\pi \pi)_{s}, P_{11}(1440) \pi$ and $D_{13^{-}}$ (1520) $\pi$ were determined 39. The PWA is compatible with known resonance properties. The largest difference to the PDG value is found for the total decay width of the $P_{13}(1720)$. From the analysis of the Roper $P_{11}$ partial wave a strong coupling of the $N(1440)$ to $N \sigma$ is suggested 40.

Due to the isoscalar $\eta$, the $\pi^{0} \eta$ final state provides selectivity to $\Delta^{*}$ intermediate states. Based on the unpolarised data, the Bonn-Gatchina PWA finds a $D_{33}$ partial wave with contributions from $\Delta(1700)$ and $\Delta(1940)$ 41. Possibly, also a further $\Delta(2350)$ adds weakly. The $\Delta(1940)$ is a highly interesting negative parity state. In quark models it would be interpreted as a radial excitation of the $\Delta(1700)$. However, the mass is unexpectedly low by about $200 \mathrm{MeV}$.

New measurements with linearly polarised photon beam show clear beam aymmetries in both the $\pi^{0} \pi^{0}$ and $\pi^{0} \eta$ channels 42,43,44. The cross section for the production of two pseudoscalar mesons can be written in the form

$$
\frac{d \sigma}{d \Omega}=\frac{d \sigma_{0}}{d \Omega}\left\{1-P_{\operatorname{lin}}\left(\Sigma \cos 2 \Phi+I^{S} \sin 2 \Phi\right)\right\} .
$$

Due to the 3-body final state, compared to Eq.1 an additional asymmtry $I^{S}$ occurs which exhibits through a $\sin 2 \Phi$ azimuthal modulation. This is however found to be compatible with zero in both final states. The $2 \pi^{0}$ asymmetries agree well with GRAAL data published for the lower energies [45.

\section{$4.5 \omega$ photoproduction}

Previous near-threshold measurements of $\omega$ photoproduction [4] had been interpreted in terms of s-channel resonances [48. However, to disentangle resonance contributions from the important t-channel exchange of $\pi^{0}$ and/or pomeron, polarisation observables are essential [49]. This motivated us to investigate the beam asymmetries in $\omega$ photoproduction using the $\omega \rightarrow \pi^{0} \gamma$ decay. In addition to the ordinary $\Sigma$ of Eq. 1, in the $\pi^{0} \gamma$ decay also the decay asymmetry $\Sigma_{\pi}$ can be accessed, which is related to the azimuthal modulation of the direction of the decay pion with respect to the photon polarisation plane. Various models predict large negative $\Sigma$ 's if s-channel resonances contribute [50]. In this case the Bonn-Gatchina PWA expects $\Sigma_{\pi} \simeq 0$. In contrast, pure t-channel mechanisms would generate $\Sigma_{\pi}= \pm 0.5$.

Experimentally the $\omega$ is clearly identified in its neutral decay yielding $3 \gamma$. However, even with the close to $4 \pi$ coverage, a number of background channels contribute significantly, mostly due to missing one photon of competing reactions with 4 final state photons, or having a splitoff from a 2 photon final state. The shaded histogram of Figure 5 shows the $\pi^{0} \gamma$ invariant mass distribution. The indicated background channels yield a reasonable MonteCarlo description of the spectrum. In the region of the $\omega$ 


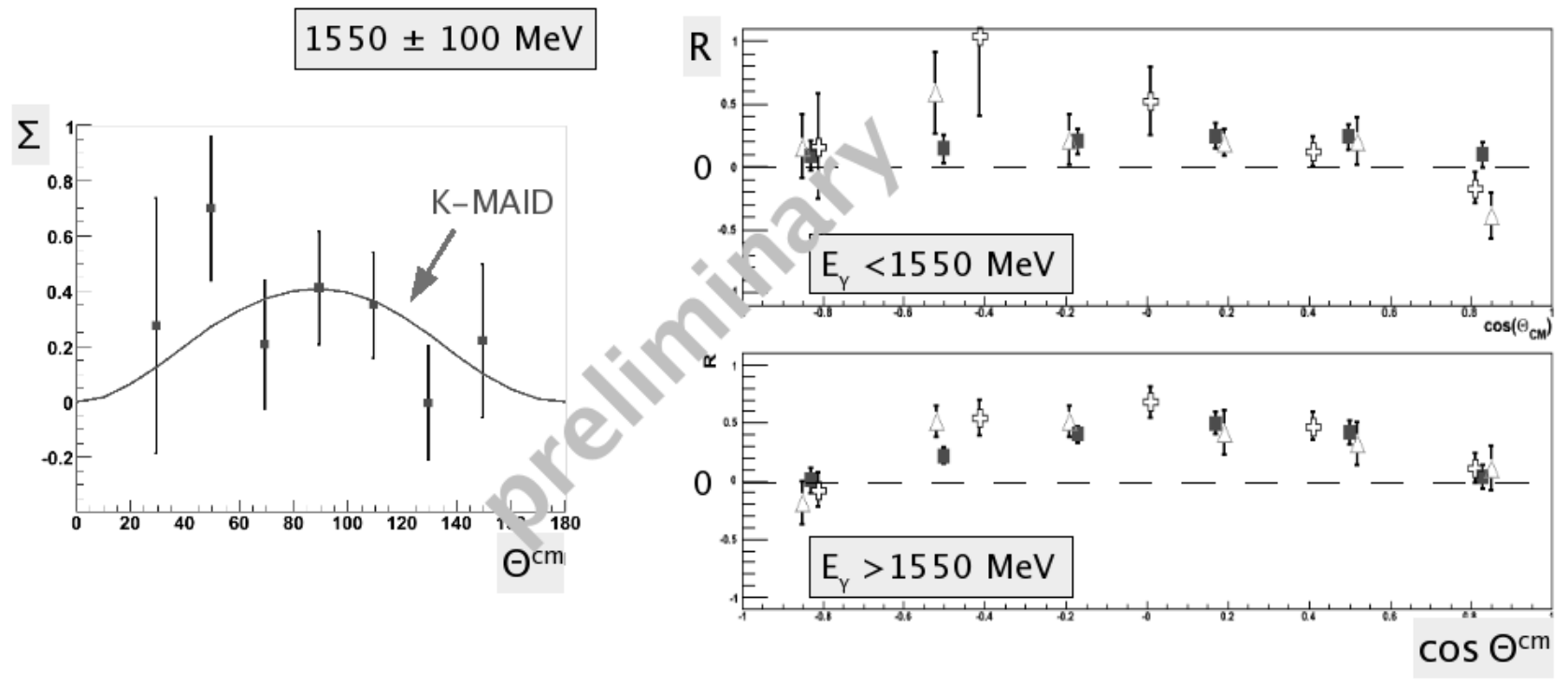

Fig. 4. Preliminary polarisation results in $K_{s}^{0} \Sigma^{+}$photoproduction off the proton [35]. Left: Photon beam asymmetry as a function of the $K \mathrm{~cm}$-angle in the energy interval $1450-1650 \mathrm{MeV}$. The errors correspond to the statistics of about a quarter of the full data set. The curve represents the K-MAID calculation. Right: Recoil hyperon polarisation in two energy intervals. The preliminary data 35] (squares) are compared to the previous measurements of Crystal Barrel/TAPS [34] (triangles) and SAPHIR 38] (crosses).

peak the far dominating part of the background is from $2 \pi^{0}$. Therefore, to subtract the background, so far binwise fits have been performed of signal plus $2 \pi^{0}$ debris to the experimental distributions. From the corresponding event numbers within $\pm 2.5 \sigma$ wide cuts around the peak the azimuthal distributions and the asymmetries were determined.

As examples, preliminary results for $\Sigma$ and $\Sigma_{\pi}$ are shown in Fig [in the bin of $E_{\gamma}=(1250 \pm 100) \mathrm{MeV}$. Good agreement is found to a recent measurement of $\Sigma$ from GRAAL 51. Compared to GRAAL the ELSA dataset extends the energy range to $E_{\gamma}=1700 \mathrm{MeV} . \Sigma_{\pi}$ (Fig. 6 bottom) is only accessible in the $\pi^{0} \gamma$ decay channel measured at ELSA. Both the large negative $\Sigma$ and the small $\Sigma_{\pi}$ seem to further support that s-channel resonances provide a substantial contribution to $\omega$ photoproduction. However, a closer characterisation of individual contributions appears premature without the measurement of further double polarisation observables, which has just started at ELSA 49.

\section{Summary and outlook}

Using the tagged photon beam of ELSA and the combined Crystal Barrel/TAPS setup several neutral meson photoproduction channels have been investigated. From the differential and total cross sections indeed indications have been found for previously unseen states. Before however unambiguous conclusions from PWA or dynamical models are possible, single and double polarisation observables need to be measured as well. As a first step, photon beam

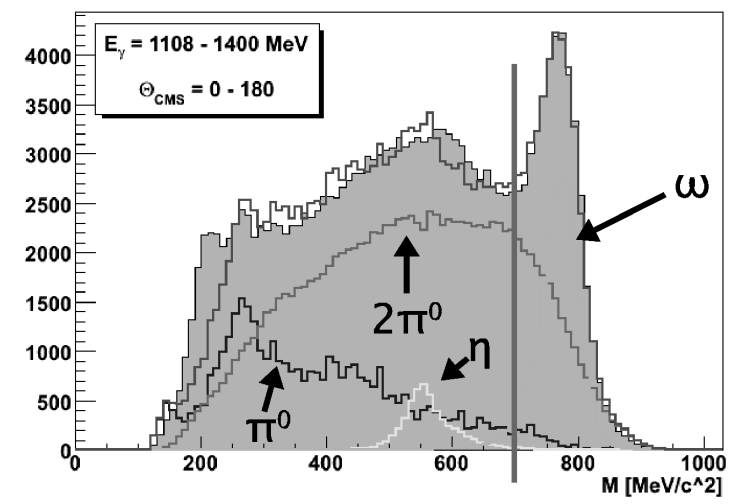

Fig. 5. $\pi^{0} \gamma$ invariant mass distribution (shaded histogram). As a result of Monte Carlo studies, the lines show the $\omega$ signal peak and main background contributions as indicated. The vertical line represents the minimum invariant mass required in the extraction of the asymmetries 46 .

asymmtries and, in the $K_{s}^{0} \Sigma^{+}$channel, recoil polarisations have been determined to further constrain the analyses. Double polarisation experiments have been prepared at ELSA and are currently underway, using the longitudinally polarised electron beam in combination with the longitudinally polarised frozen spin target. Similar plans are pursued at several other laboratories. The double polarisation experiments are expected to nail down the question of the existence of at least some of the newly found states. 

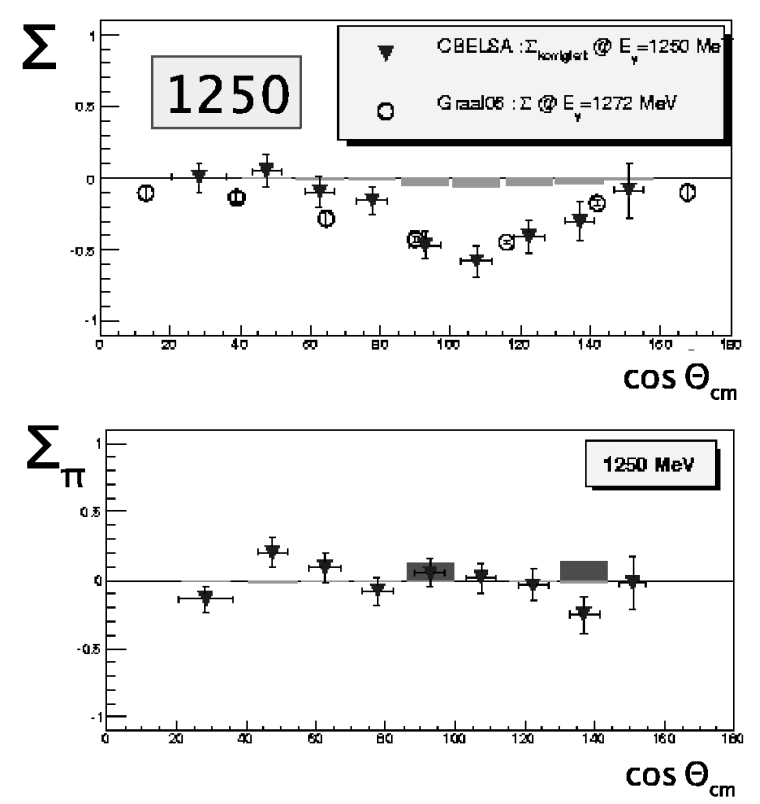

Fig. 6. Preliminary results (filled triangles) of the beam asymmetry (upper diagram), $\Sigma$, and the decay asymmetry, $\Sigma_{\pi}$ (lower diagram) for the energy bin $E_{\gamma}=1250 \pm 100 \mathrm{MeV}$. GRAAL data (open circles) of $\Sigma$ are shown for comparison. Purely statistical errors are attached to the data points, the bars represent estimates of the systematic uncertainties.

I am grateful to the doctoral students who provided their results for my talk prior to publication. In particular I like to thank Kathrin Fornet-Ponse, Holger Eberhardt, Susanne Kammer, Andre Süle, Frank Klein, Ralf Ewald, Ralph Castelijns, Eric Gutz, Michael Fuchs, Vahe Sokhoyan, Igal Jägle. The discussions with my colleagues at the Helmholtz Institute für Strahlen- und Kernphysik and at the Physikalisches Institut, B. Bantes, R. Beck, D. Elsner, J. Hannappel, V. Kleber, Fritz Klein, E. Klempt, and U. Thoma, provide a constant source of inspiration for me. I also participated a lot of the expertise of B. Krusche from Basel. The enthusiasm of the accelerator group was benefitial to all experiments; a special thank goes to W. Hillert. Unthinkable without H. Dutz are the target polarisation experiments.

The investigations at ELSA are supported by the federal state of North-Rhine Westphalia and by the Deutsche Forschungsgemeinschaft within the SFB/TR-16.

\section{References}

1. see e.g. S. Capstick and W. Roberts, Prog. Part. Nucl. Phys. 45 (2000) 241

2. U. Löring et al., Eur. Phys. J. A10 (2001) 395, 447

3. A.C. Kalloniatis, D.B. Leinweber, A.G. Williams (Eds.), "Lattice Hadron Physics", Lecture Notes in Physics 663, Springer (2005)

4. S. Capstick and W. Roberts, Phys. Rev. D 49 (1994) 4570

5. V. Crede et al., Phys. Rev. Lett. 94 (2005) 012004
6. B. Saghai and Z. Li, Proceedings of the Workshop on the Physics of excited Nucleons, Pittsburgh (2002), ed. by S.A. Dytman and E.S. Swanson (World Scientific, 2003), p. 166

7. G.Y. Chen et al., Nucl. Phys. A723 (2003) 447

8. W.-T. Chiang and F. Tabakin, Phys. Rev. C55 (1997) 2054

9. G. Knöchlein, D. Drechsel and L. Tiator, Z. Phys. A352 (1995) 327

10. see e.g. V. Burkert, these proceedings

11. W. Roberts, Proceedings of the NSTAR'05 (Tallahassee) (S. Capstick, V. Crede, P. Eugenio edts., World Scientific 2005) p. 138.

12. E. Aker et al., Nucl. Instr. Meth. A321 (1992) 69

13. R. Novotny et al., IEEE transaction on nuclear science $\mathbf{3 8}$ (1991) 378

14. W. Hillert, Eur. Phys. J. A28, s01 (2006) 139

15. D. Elsner et al., Eur. Phys. J. A33 (2007) 147

16. D. Elsner, these proceedings

17. G. Suft et al., Nucl. Instr. Meth. A538 (2005) 416

18. A.R. Gabler et al., Nucl. Instr. Meth. A346 (1994) 168

19. O. Bartholomy et al., Eur. Phys. J. A33 (2007) 133

20. M. Dugger et al., Phys. Rev. Lett. 89 (2002) 222002 and 249904(E)

21. A. Süle, doctoral thesis, Bonn (in preparation)

22. V. Kouznetsov et al., PIN Newsletter 16 (2002) 160

23. O. Bartalini et al., arXiv:0707.1385 1[nucl-ex]

24. W.T. Chiang, S.N. Yang, L. Tiator, D. Drechsel, Nucl. Phys. A700 (2002) 429;

see also http://www.kph.uni-mainz.de/MAID/maid.html

25. A.V. Anisovich et al., Eur. Phys. J. A25 (2005) 427

26. see e.g. A. Kouznetsov, these proceedings

27. I. Jägle, doctoral thesis, Basel (in preparation)

28. see e.g. B. Krusche et al., proposal ELSA/3-2005

29. see e.g. A. Fix, L. Tiator, and M. V. Polyakov, nucl-th/0702034

30. A. Anisovich, these proceedings

31. V. Shklyar, these proceedings

32. R. Bijker, F. Iachello, and A. Leviathan, Phys. Rev. D 55 (1997) 2862

33. S. Capstick and W. Roberts, Phys. Rev. D 58 (1998) 074011

34. R. Castelijns et al., nucl-ex/0702033

35. R. Ewald, doctoral thesis, Bonn (in preparation)

36. A. Sarantsev et al., Eur. Phys. J. A25 (2005) 441

37. A. Usov and O. Scholten, Phys. Rev. C 72 (2005) 025205

38. R. Lawall et al., Eur. Phys. J. A24 (2005) 275

39. U. Thoma et al., submitted to Phys. Lett. B

40. A. Sarantsev et al., submitted to Phys. Lett. B

41. I. Horn et al., submitted to Phys. Rev. Lett.

42. E. Gutz, doctoral thesis, Bonn (in preparation)

43. V. Sokhoyan, doctoral thesis, Bonn (in preparation)

44. E. Gutz, these proceedings

45. Y. Assafiri et al., Phys. Rev. Lett. 90 (2003) 222001

46. F. Klein, doctoral thesis, Bonn (in preparation)

47. J. Barth et al., Eur. Phys. Journal A 18 (2003) 117

48. S. Penner et al., Phys. Rev. C 66 (2002) 055212, V. Shklyar et al., Phys. Rev. C 71 (2005) 055206 and C 72 (2005) 019903

49. H. Schmieden et al., et al., proposal ELSA/4-2005

50. see e.g. A. I. Titov and T.-S. H. Lee, Phys. Rev. C 66 (2002) 015204, and Q. Zhao, J.S. Al-Khalili and P. L. Cole, Phys. Rev. C 71 (2005) 054004

51. J. Ajaka et al., Phys. Rev. Lett. 96 (2006) 132003 
